\title{
AwkwardForth: accelerating Uproot with an internal DSL
}

\author{
Jim Pivarski ${ }^{1, *}$, Ianna Osborne ${ }^{1, * *}$, Pratyush Das $^{2, * * *}$, David Lange ${ }^{1, * * * *}$, and Peter Elmer ${ }^{1, \dagger}$ \\ ${ }^{1}$ Princeton University, Princeton, New Jersey, United States. \\ ${ }^{2}$ Institute of Engineering and Management, Kolkata, West Bengal, India.
}

\begin{abstract}
File formats for generic data structures, such as ROOT, Avro, and Parquet, pose a problem for deserialization: it must be fast, but its code depends on the type of the data structure, not known at compile-time. Just-in-time compilation can satisfy both constraints, but we propose a more portable solution: specialized virtual machines. AwkwardForth is a Forth-driven virtual machine for deserializing data into Awkward Arrays. As a language, it is not intended for humans to write, but it loosens the coupling between Uproot and Awkward Array. AwkwardForth programs for deserializing record-oriented formats (ROOT and Avro) are about as fast as C++ ROOT and 10-80× faster than fastavro. Columnar formats (simple TTrees, RNTuple, and Parquet) only require specialization to interpret metadata and are therefore faster with precompiled code.
\end{abstract}

\section{Motivation}

Despite being written in Python, Uproot [1] can read simple data types from ROOT TTrees [2] as fast as any precompiled code because the values are already contiguous in a raw view of the file. No computations are required to get a ROOT TBasket of numerical data from disk into memory, as an array, except to swap endianness if little-endian arrays are desired. Python's NumPy [3] library casts data from raw bytes as a metadata-only operation, and performs operations that scale as $O(n)$, where $n$ is the length of the array, in precompiled code.

For more complex data types, however, the cost of computing in Python increases. Variable-length (“jagged”) arrays are relatively quick, since ROOT's TTree format separates the numerical content of these arrays from the integers that define the starting positions of each entry's array. This is nearly the format required by Awkward Array [4], and only needs minor arithmetic transformations. Objects with fixed-size headers and consisting of fixed-size fields, such as std: : vector $<$ TLorentzVector $>$, can be extracted using NumPy tricks, though these tricks require more intermediate arrays, further slowing the transformation. Finally, general objects with nested, variable-length data, the simplest of which is std: : vector $<$ std: : vector $<$ float $>>$, require custom code to parse each data type. In Python, such code is hundreds of times slower than the equivalent $\mathrm{C}++$.

Previously, we quantified this slow-down [5] using ROOT TTrees containing float, std: : vector $<$ float $>$, and vectors of vectors up to three levels deep, reading them with

\footnotetext{
*e-mail: pivarski@princeton.edu

**e-mail: ianna.osborne@cern.ch

***e-mail: reikdas@gmail.com

****e-mail: david.lange@ cern.ch

†e-mail: peter.elmer@cern.ch
} 
the then-current Python codebase and with custom $\mathrm{C}++$ code, which represents what is possible now that Awkward Array is implemented in $\mathrm{C}++$. The read performance of float data is identical for Python and $\mathrm{C}++$, the $\mathrm{C}++$ is several times faster for $\mathrm{std}$ : : vector $<$ float $>$, and the gap widens to factors of hundreds for the doubly-nested and triply-nested cases. Leveraging Awkward Array's C++ layer to accelerate Uproot is a worthwhile goal.

However, unless we limit our attention to special cases like nested vectors of numbers, this deserialization code is not known at compile-time. The byte-for-byte layout of a complex data type is expressed as data, in the TStreamerInfo of a ROOT file, and is therefore discovered at runtime. Moreover, the specifics of ROOT deserialization should not be spread between two packages, Uproot and Awkward Array: Uproot should focus entirely on ROOT I/O as Awkward Array focuses on array manipulation. The problem is to satisfy three constraints:

1. Deserialization code must not be hundreds of times slower than compiled code.

2. This code must be generated at runtime from TStreamerInfo.

3. All "knowledge" of ROOT I/O must be in Uproot, not Awkward Array.

The first two constraints could be satisfied by just-in-time (JIT) compilation. This is, in fact, what ROOT's Cling compiler [6] does. The third constraint could be satisfied by adding a $\mathrm{C}++$ layer to Uproot. Both of these solutions, however, would significantly complicate the distribution of the Uproot or Awkward Array packages, and portability is a high priority.

This paper describes a different solution to the constraints listed above, which does not affect the portability of Uproot or Awkward Array. We introduce AwkwardForth, a domainspecific language (DSL) for deserializing arbitrary data into Awkward Arrays that is runtimeinterpreted but nearly as fast as compiled code. Unlike DSLs intended for humans to read and write, this DSL is "internal," only used to communicate between software packages. Uproot's task becomes one of expressing ROOT I/O logic in AwkwardForth and Awkward Array executes it, returning filled arrays.

\section{AwkwardForth}

AwkwardForth is a member of the Forth family of languages, which includes Postscript [7], another internal DSL. AwkwardForth adheres to a subset of the ANSI Forth Standard [8] and has extensions for interpreting arbitrary input buffers and filling columns for Awkward Array.

Like all Forths, AwkwardForth is primarily concerned with stack manipulation. The runtime environment features a stack of integers and programs define "words" that manipulate the stack (as well as input and output buffers, in the case of AwkwardForth). Each word consumes and produces an arbitrary number of arguments and return values through this stack, and hence Forth words are more general than functions in a typical programming language. Whereas functional programming languages eliminate or minimize side-effects, Forth acts exclusively through side-effects. As such, it is more like an extensible assembly language.

The popularity of Forth peaked in the early 1980's because its interactive interpreter could fit within the tight resource constraints of early personal computers [9]. This same interactive minimalism makes it an ideal candidate for running fast programs that must be generated at runtime, such as deserializing ROOT files. On a 2.2 GHz CPU core, AwkwardForth takes about $5 \mathrm{~ns}$ to evaluate each word, compared to about $900 \mathrm{~ns}$ for Python to evaluate a bytecode.

Though motivated by the problem of ROOT deserialization, AwkwardForth is intended for the general problem of deserializing non-columnar data formats into columnar Awkward Arrays. Many file formats, such as ProtoBuf [10], Thrift [11], Avro [12], FlatBuffers [13], and JSON [14], describe data structures in a record-oriented layout, with all fields of one record 
contiguous with each other, while columnar formats like simple TTrees, RNTuple [15], Parquet [16], Arrow [17], and Awkward Array place all values of one field contiguous with each other before moving on to the next field. Conversions between columnar formats can be very fast, sometimes casting, rather than copying, the columns. Record-oriented formats, on the other hand, must be fully parsed. In this paper, we examine AwkwardForth's deserialization performance for ROOT TTrees (columnar and record-oriented), Avro, and Parquet.

Awkward Array has a tool for converting arbitrary record-oriented data into Awkward Arrays: ArrayBuilder constructs arrays in an append-only order, driven by commands such as begin/end_record, switch to field, append integer, and begin/end_list. A consequence of this generality is that ArrayBuilder discovers the array's data type at runtime, adding output buffers as the observed type gets more complex. While this is great for JSON, type discovery is unnecessarily slow for formats whose type is known in advance, though perhaps not as early as compile-time. (Uproot currently uses ArrayBuilder in Python.) In this paper, we also present the design of a TypedArrayBuilder, which generates AwkwardForth programs from data types, but is still driven by ArrayBuilder-like commands.

\section{AwkwardForth virtual machine}

AwkwardForth is implemented in $\mathrm{C}++$ as a virtual machine with byte-compiled instructions. It is not interactive, unlike most Forths, since it is intended to be programmed algorithmically. Even the TypedArrayBuilder use-case works by "wiring" its fixed suite of commands to algorithmically generated Forth subroutines. Some TypedArrayBuilder commands must change the state of its finite-state machine: for instance, when filling an array of doubly nested lists of integers like $[[1,2],[3]],[],[[4],[5]]$, the first begin_list ([) puts it into a state that expects another begin_list ([) or end_list (]); the second puts it into a state that expects integer or end_list (]). And yet, each of these commands must return control-flow to its caller and remember its state for the next call. TypedArrayBuilder's AwkwardForth machine must be able to stop and resume with its state intact (unlike most Forths). AwkwardForth, therefore, has built-in words to control its own execution (pause and halt), and the execution may be resumed from outside the machine.

When an AwkwardForth machine is first constructed, it compiles its source code (text) into bytecode instructions (variable-length sequences of 32-bit integers-a jagged array), so that they can be interpreted more quickly. This is the same sense in which Python code is "compiled." Built-in words translate to 1-3 integer codes, the second and third being arguments that modify the first. User-defined words are separate sequences of instructions, called a "dictionary" in Forth. Control-flow structures, such as if and loop, are implemented as unnamed user-defined words so that their bodies are fixed-width "calls" into the dictionary, simplifying the logic of instruction pointer manipulation.

All errors are caught in the compilation phase except for 10 possible runtime errors: "user halt," "recursion depth exceeded," "stack underflow," "stack overflow," "division by zero," "read beyond," "seek beyond," "skip beyond," "rewind beyond," and "varint too big." The last 5 of these are specific to parsing input buffers.

All runtime execution is implemented in a single "noexcept" $\mathrm{C}++$ function for speed.

An arbitrary number of named input buffers and named output buffers are associated with each AwkwardForth program. Inputs must be supplied at the beginning of a run; outputs are also created at this time. Input buffers, which are the data to be parsed, are viewed as untyped raw bytes, interpreted by the words of the program itself. Output buffers, which are columns to use in a new Awkward Array, have specific numerical types and can only be filled with that type. Inputs have a fixed size and are seekable; outputs grow in an append-only way. 


\section{AwkwardForth language}

Like all Forth languages, AwkwardForth has an extremely simple syntax: whitespace delimits tokens and tokens are interpreted in reverse Polish order: "3 4 +" means the sum of 3 and 4 (the first two words put numbers on the stack and the third pops them and pushes their sum).

AwkwardForth has 57 standard built-in words and 61 extensions for input and output buffers, though 50 of the 61 are different ways of interpreting the bytes of an input buffer. Two special words control the state of the virtual machine: pause and halt, and three do not generate runtime code at all: they declare variables, inputs, and outputs.

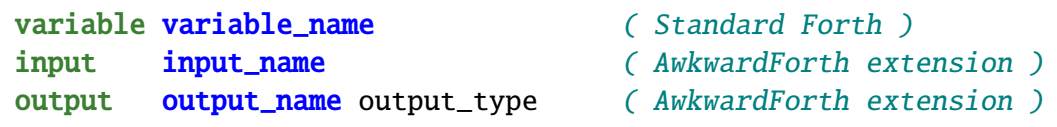

As in Standard Forth, user-defined words are bracketed between : and ; and the main code is anything outside these definitions. Control structures are pairs of words like if-then and do-loop, or triples like if-else-then and begin-while-repeat.

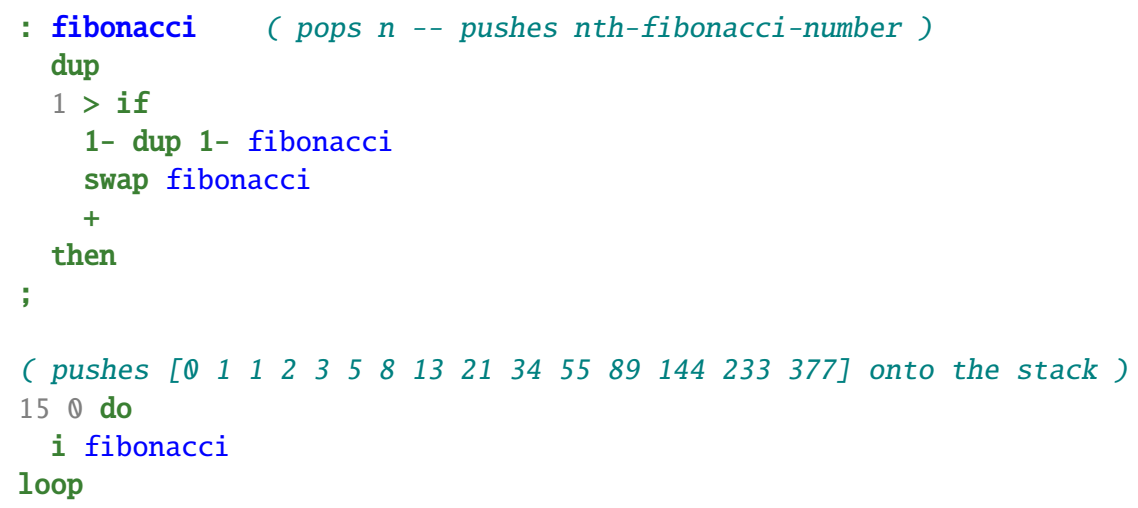

The 50 special words for parsing are all arrows with a type code, an optional ! for bigendian and an optional \# to pull a number off the stack to determine how many times to repeat it. Thus, "input_name i-> output_name" reads 4 bytes from the input as an integer and writes it to the output, "input_name !d-> output_name" reads 8 bytes as a big-endian, double-precision float, and "100 input_name \#I-> output_name" reads 100 unsigned 4byte integers. In any case, the destination may be the stack: "input_name i-> stack".

The type codes are taken from Python's struct module, which AwkwardForth most closely resembles in purpose (but vastly exceeds in expressiveness). Two special codes, varint-> and zigzag->, read variable-length unsigned integers and zig-zag encoded signed integers, which are used in many file formats, including Avro and Parquet. Also, Parquet needs a command to read $n$-bit unsigned integers, hence 2 bit->, 3bit->, etc. for any $n$.

Outputs are similar, but less varied because they have preassigned types. They may be filled directly from an input (bypassing the stack for efficiency and to preserve the types of floating point numbers) or filled from the stack: "output_name <- stack". A shortcut for appending the last output plus a value from the stack is "output_name $+<-$ stack".

More built-in words can be added to handle problems posed by input formats. Since each built-in word has a fixed $5 \mathrm{~ns}$ cost, specialized words result in faster AwkwardForth code.

\section{AwkwardForth programs for ROOT, Avro, and Parquet}

To read a TBasket of std: : vector<std: : vector $<$ float $>>$ from ROOT, we use the following AwkwardForth program: 


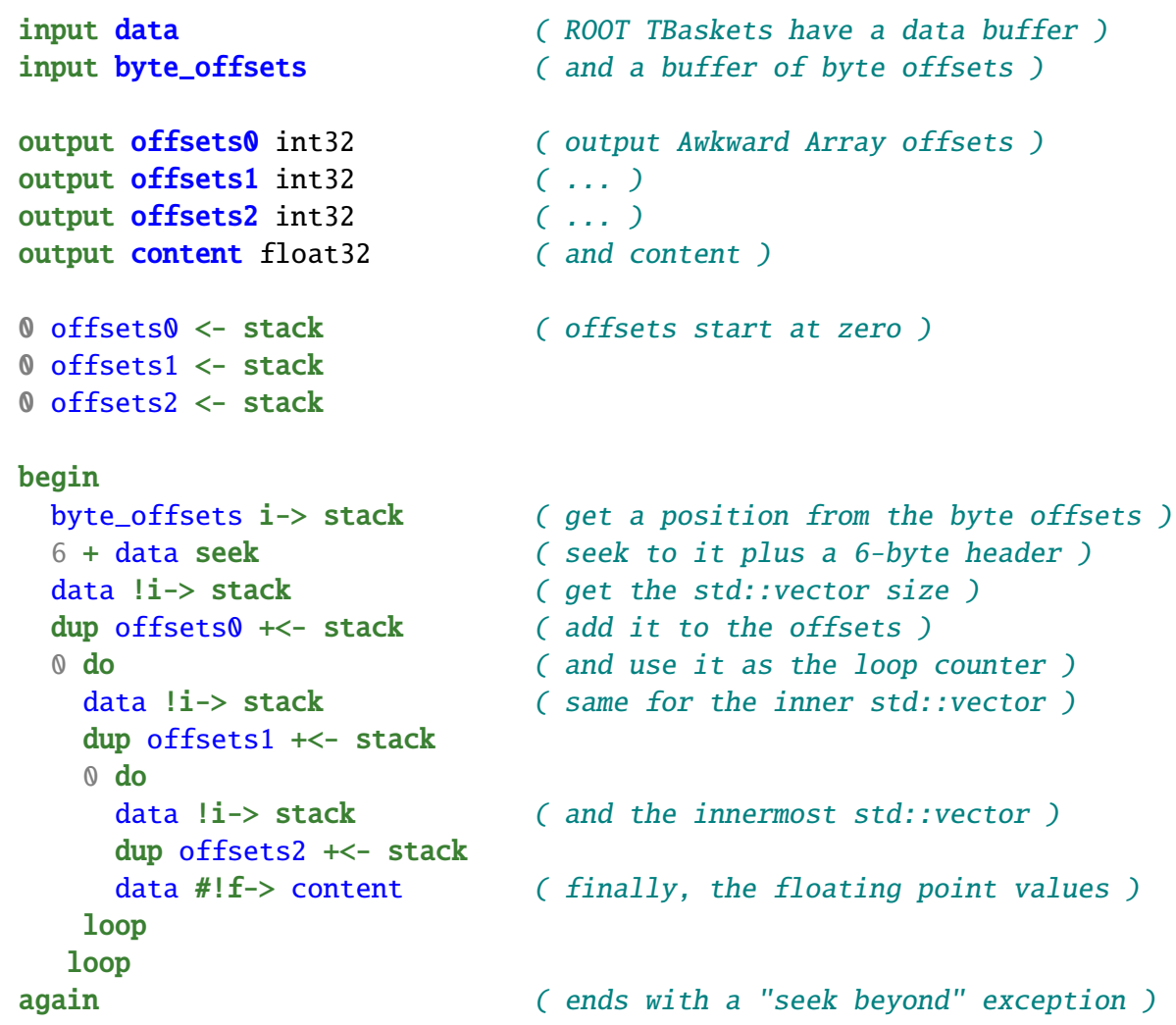

To read data with the same structure from Avro, we use the program below. It is applied to each data block of an Avro container file, with the AwkwardForth machine stack initialized with the number of entries in the block.

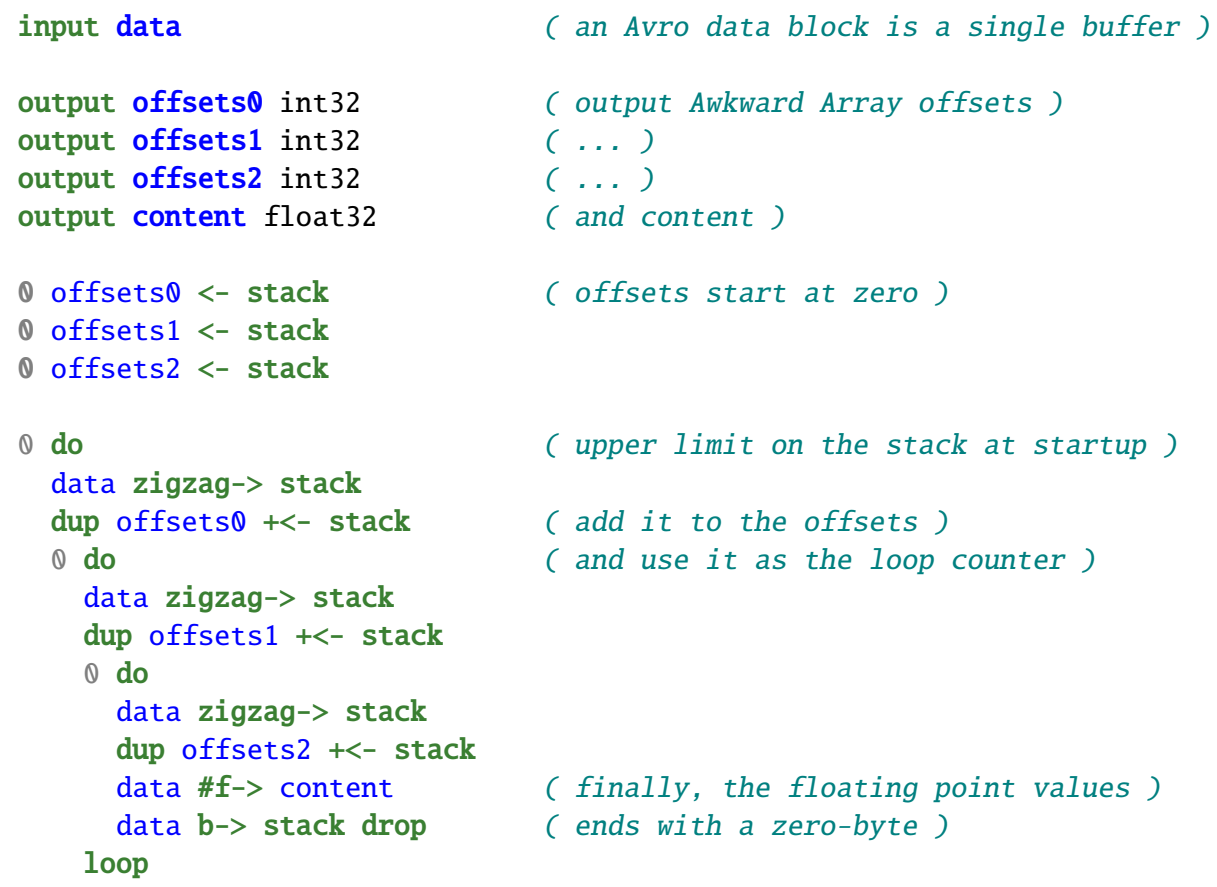




$$
\begin{array}{ll}
\text { data b-> stack drop } & \text { ( lists also end with a zero-byte ) } \\
\text { loop } & \text { ( lists also end with a zero-byte) } \\
\text { data b-> stack drop } & \\
\text { loop } &
\end{array}
$$

Parquet is a columnar file format, so the floating-point content comes in a form that's ready to use in Awkward Array, without even copying the uncompressed buffer. However, the nested list structure is in a highly packed form: repetition levels indicating the depth of each floating-point item, which are further run-length encoded or bit-packed in small groups.

We unpack them using two AwkwardForth programs: the first produces the repetition levels as unsigned 1-byte integers and the second converts them into three levels of offsets.

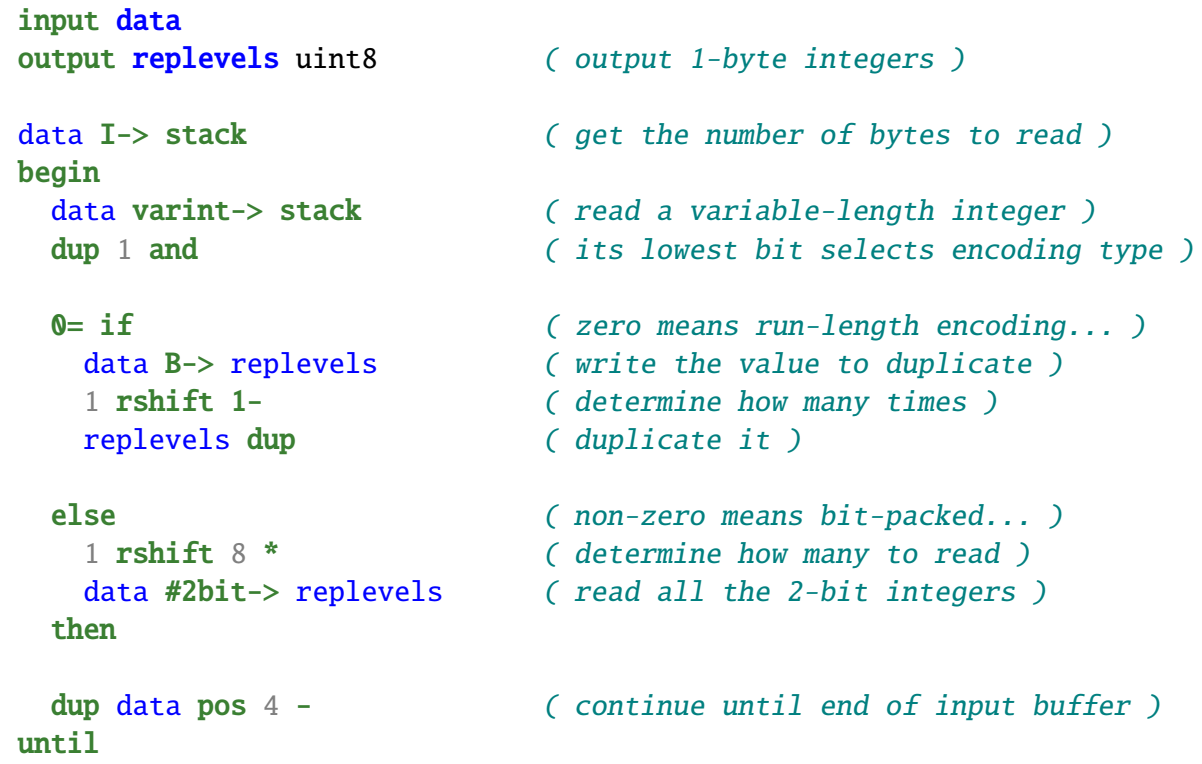

This last program interprets the repetition levels as offsets. It uses variables to count the number of items at each level of list depth since all three need to be increased concurrently and using swap or rot to manage them on the stack would be unnecessarily complex. The Standard Forth words @, !, and +! read, write, and increment an off-stack variable. Variables have the same integer type as the stack and are initially zero.

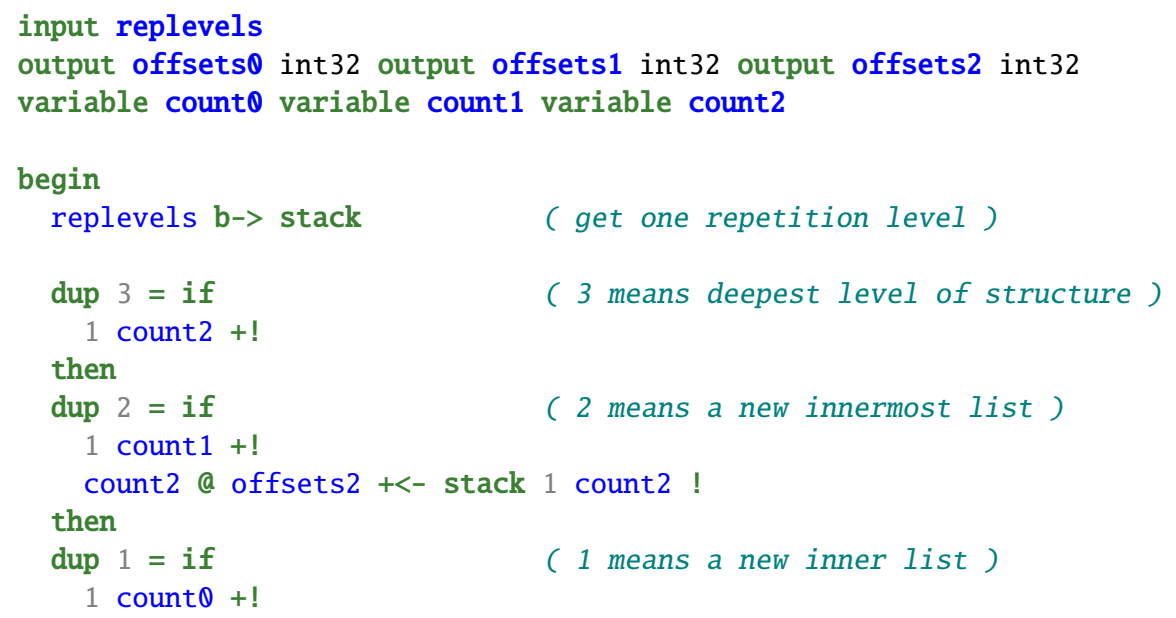




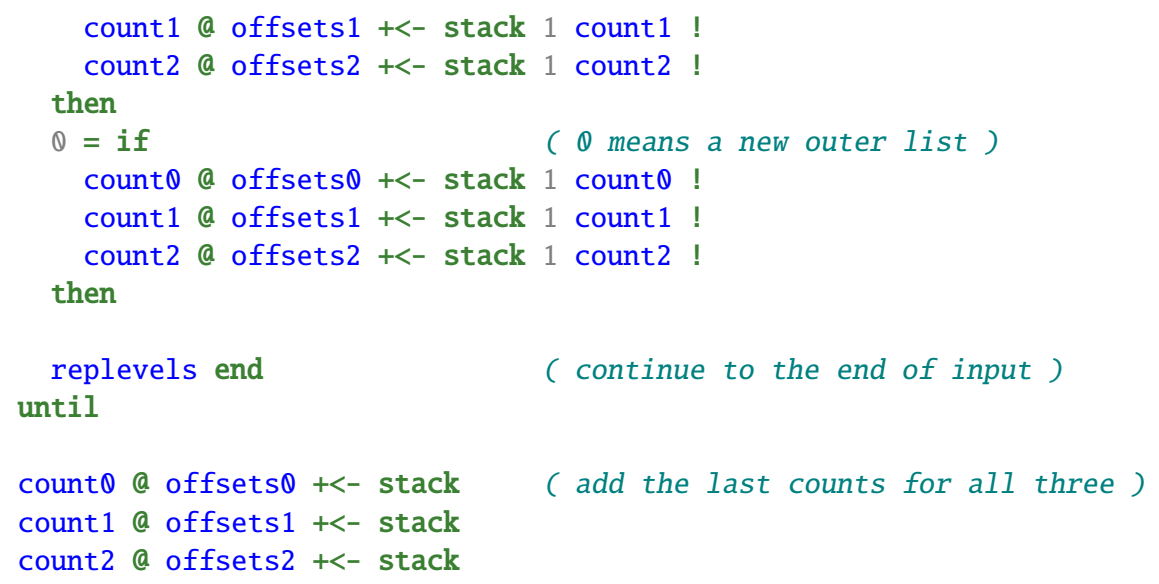

Programs such as these would not be written (and commented!) by hand, but generated by Uproot, other file-readers that produce Awkward Arrays, or TypedArrayBuilder.

\section{Example of an AwkwardForth program for TypedArrayBuilder}

TypedArrayBuilder was implemented using AwkwardForth. It takes an input type and generates a machine that fills that type, pausing before each input command. Commands are represented by enumeration constants, and the program flow depends on the sequence of commands it receives. The example program below accepts a triply nested list of float.

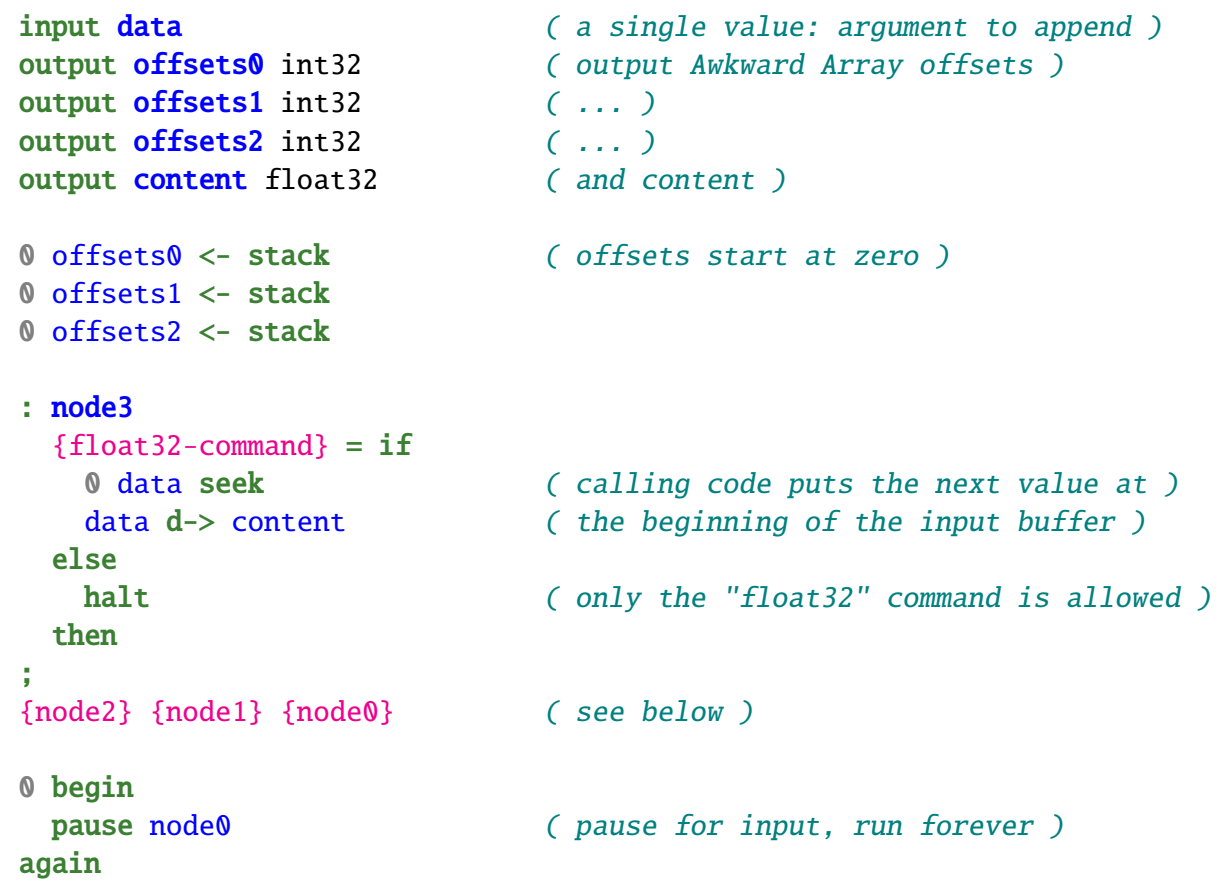

The words in curly brackets are strings to be replaced, such as the following for $\{$ nodeN $\}$ :

: \{node_name\}

\{begin_list-command\} $<$ if ("begin_list" is required here) halt

then 


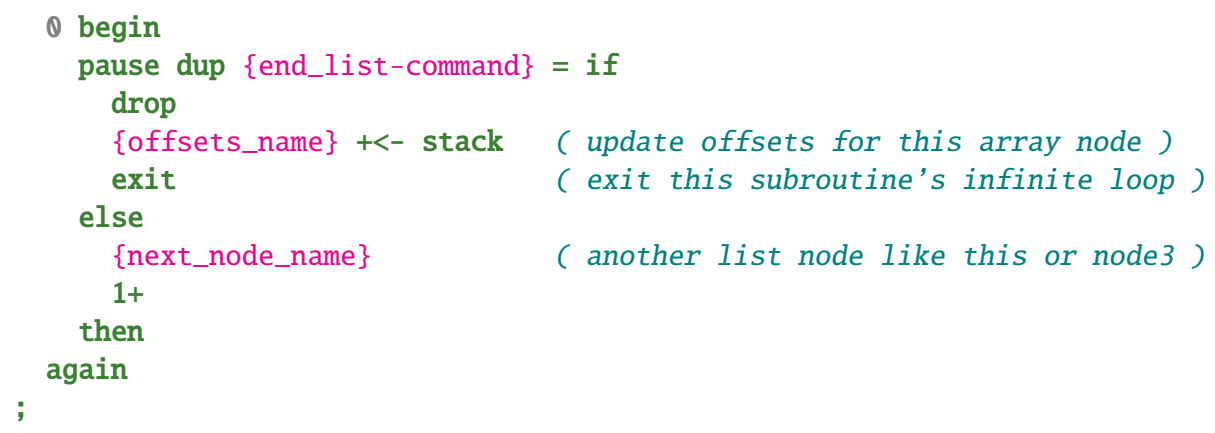

The $\{*$-command $\}$ substitutions are the enumeration constants. The virtual machine waits at a pause word until TypedArrayBuilder puts a command number on the stack (and a value in the input buffer for the float 32 command), then resumes program flow, letting AwkwardForth format the output or halt if the command is not allowed. Thus, TypedArrayBuilder itself can be statically compiled but "wired" to different actions at runtime.

\section{Performance}

Figure 1 presents single-threaded deserialization rates of uncompressed ROOT, Avro, and Parquet files from a warmed filesystem cache on an Intel i7-8750H $(2.2 \mathrm{GHz})$ processor. There are four test files for each format: 4-byte float, variable-length lists of float, doubly nested lists of lists of float, and triply nested lists of lists of lists of float. For ROOT files, variable-length lists are std: : vector, but in Avro they are called "arrays" (despite being variable-length), and in Parquet, they are called "repeated groups." All four types of files contain exactly 1073741824 float values and are all approximately 4 GiB (large, but well within the $15.5 \mathrm{GiB}$ of physical RAM). The lengths of the lists at each level of depth are Poisson-distributed with a mean of 8.0 items per list. ROOT's TBasket size, Avro's data block size, and Parquet's row-group and page sizes were all fixed at $64 \mathrm{MiB}$.

The ROOT files were read in three ways: (1) using C++ ROOT, loading data with TBranch: : GetEntry) and copying it into offsets $\theta$, offsets 1 , offsets 2 , and content buffers for Awkward Array, (2) using Uproot 4.0.1's Python code, and (3) using AwkwardForth (with Uproot to find the TBaskets within the file).

As an additional comparison, the same data were converted into ROOT's future RNTuple format, which is truly columnar. As expected (reproducing our previous results [5]), float data are fastest to read, being essentially a memory-copy from TBaskets or RNTuple pages into the content buffer. (ROOT's TBranch: : GetEntry is not as fast as direct memory access, which could be enabled by switching to ROOT's BulkIO feature, but we did not attempt that in this study.) RNTuple maintains this speed for all levels of nestedness for the same reason, while Uproot's NumPy tricks slow down reading of std: : vector $<$ float $>$ and Uproot's pure Python is orders of magnitude slower for any deeper nesting. However, AwkwardForth keeps pace with ROOT's TBranch: : GetEntry at all levels of nestedness.

We next compared AwkwardForth with fastavro, the leading Python package for reading Avro files. fastavro is a Python extension library, using the $\mathrm{C}$ Avro implementation for speed. However, fastavro is compiled without knowledge of the schemas of the Avro files, which limits this advantage. AwkwardForth, on the other hand, has specialized Forth code for each data type and it's fast enough to read Avro 10-80× faster than fastavro.

Finally, we compared AwkwardForth with pyarrow, a Python extension library for the $\mathrm{C}++$ Arrow and Parquet projects. In this case, pyarrow outperforms AwkwardForth by factors of 1.5-8×. The AwkwardForth programs for Parquet in Section 5 show why: there's nothing about them that specializes to the data type except for the \#2bit-> repetition level reader 


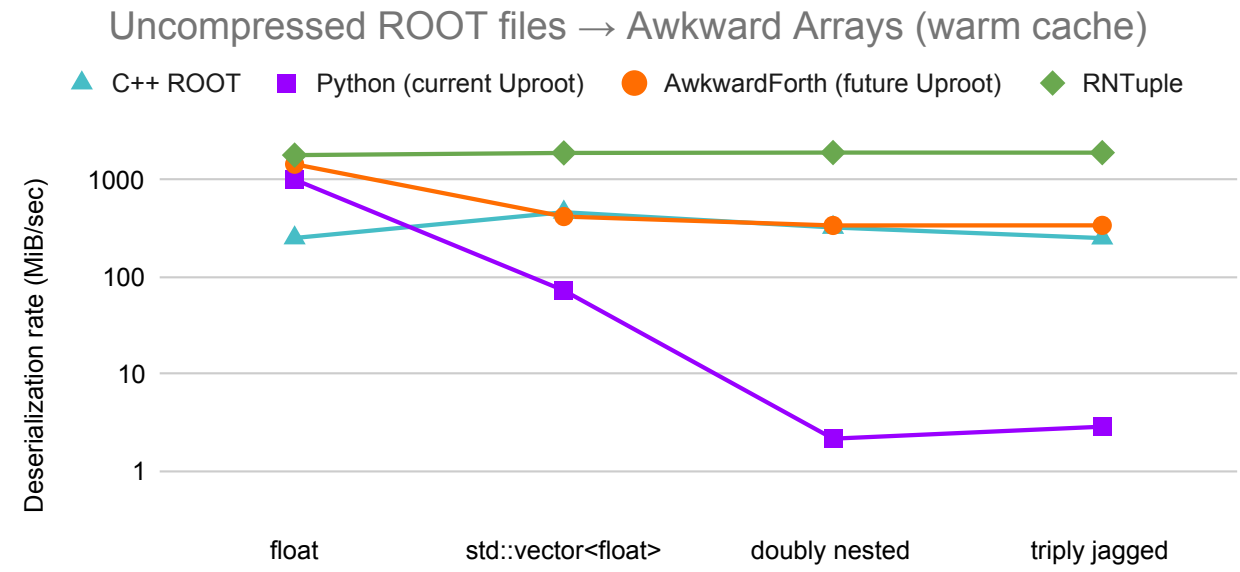

\section{Uncompressed Avro files $\rightarrow$ Awkward Arrays (warm cache) \\ $\Delta$ fastavro AwkwardForth}

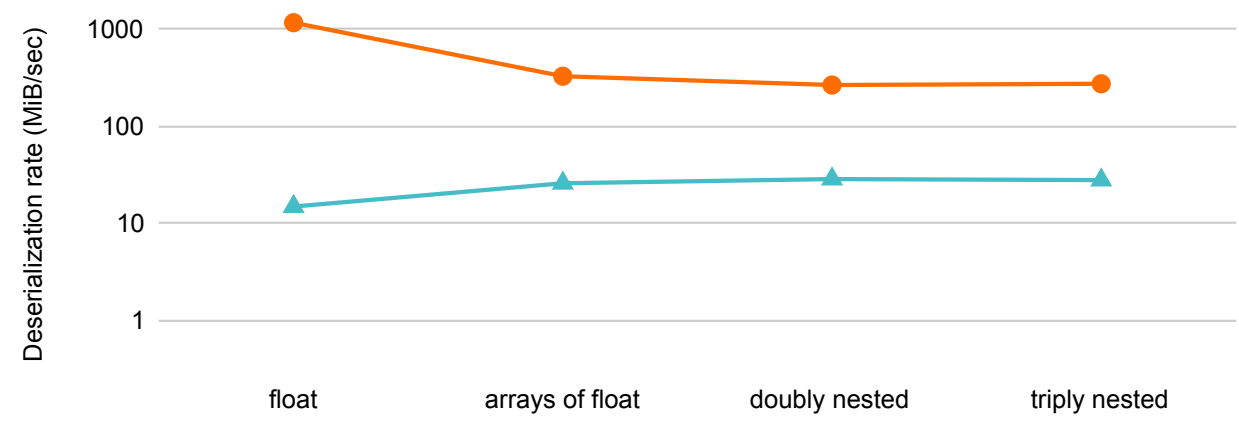

\section{Uncompressed Parquet files $\rightarrow$ Awkward Arrays (warm cache)}

$\Delta$ pyarrow AwkwardForth

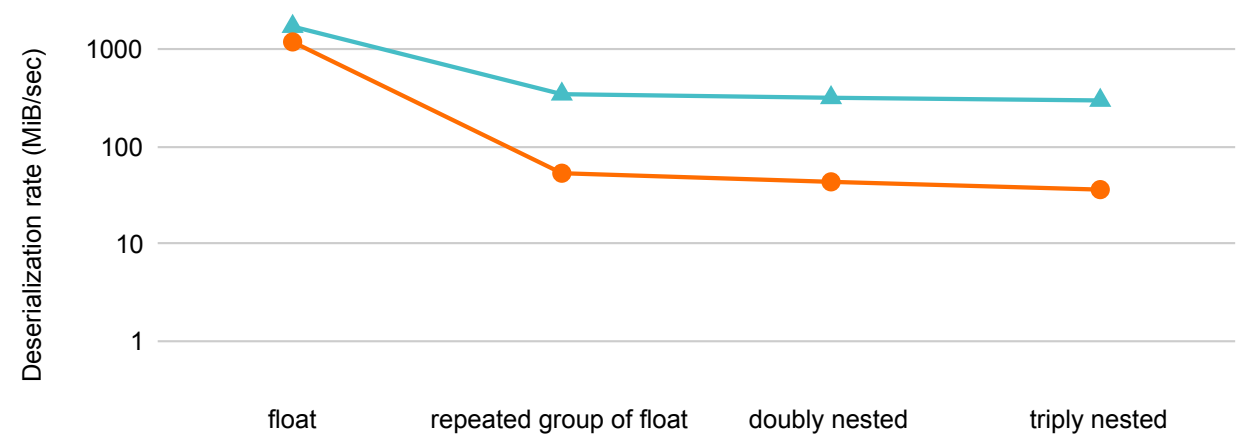

Figure 1. Deserialization rate for converting uncompressed ROOT, Avro, and Parquet files to Awkward Arrays from warm cache. $\mathrm{C}++\mathrm{ROOT}$, fastavro, and pyarrow are the leading libraries for each file type. 

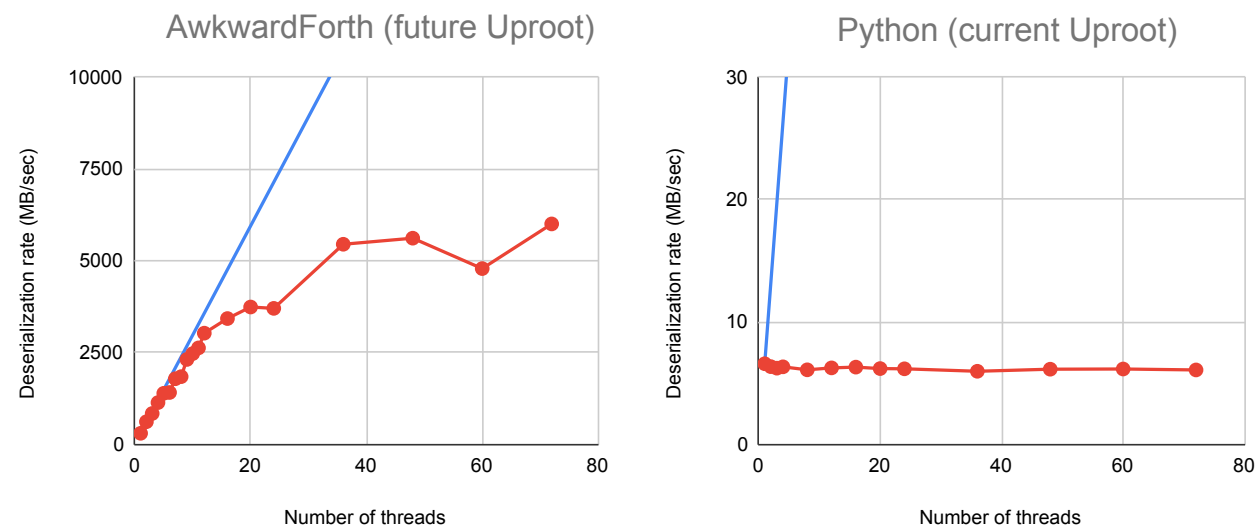

Figure 2. Scaling of deserialization by the number of threads. AwkwardForth (when called from Python) releases the Python GIL, which otherwise prevents any gains from parallel processing.

and the number of count variables and offset buffers. Any data type can be efficiently read with the same, unspecialized $\mathrm{C}++$ code. This is true in general of columnar data formats, including RNTuple, so we have no plans of applying AwkwardForth to read columnar data.

For compressed data (not shown in Figure 1), all rates are suppressed by a constant for each algorithm. LZ4 is about $10 \%$ slower than the memory copy, but ZLIB is $10 \times$ slower than the memory copy, so AwkwardForth is not the bottleneck for ZLIB-compressed data.

We also studied the scaling of AwkwardForth with threads. The AwkwardForth virtual machine is stateful, but lightweight: many can be launched at once, one for each thread. Figure 2 shows deserialization rate of the ROOT files on an AWS c5.18xlarge instance, which has 72 cores. Linear scaling falls off at about 20 threads, or 4-5 GiB per second, which may be a limitation of memory bus supplying data from RAM. Python, by comparison, does not scale at all because of its Global Interpreter Lock (GIL) [18].

All scripts used to produce Figures 1 and 2 are available on GitHub [19].

\section{Conclusions}

AwkwardForth is a "lightweight" solution to the problem of generating fast deserialization code whose form is only known at runtime. In all, it consists of 3388 lines of $\mathrm{C}++$ code in Awkward Array (version 1.1.0), with no dependencies. Unlike user-facing DSLs, its syntax is not subject to usability constraints, only ease of algorithmic generation and speed. As shown in Section 7, it keeps pace with ROOT's own TTree deserialization, exceeds fastavro for Avro, and is slower than but within an order of magnitude of pyarrow for Parquet.

Most importantly, AwkwardForth vastly improves upon the Python code in Uproot, speeding up std: : vector $<$ std: : vector $<$ float $>>$ deserialization by factors of hundreds, and it makes parallel TBasket deserialization worthwhile. To integrate AwkwardForth into Uproot, every function that generates deserialization code in Python must be supplanted by a function to generate AwkwardForth. This can be a gradual transition, as missing Forth-generators can fall back to Python. We expect the majority of data types to be completed by the end of 2021 .

\section{Acknowledgements}

This work was supported by the National Science Foundation under Cooperative Agreement OAC-1836650 (IRIS-HEP). 


\section{References}

[1] J. Pivarski et al., Uproot, https://doi .org/10.5281/zenodo. 4543730

[2] R. Brun, F. Rademakers, ROOT: An object oriented data analysis framework, Nucl. Instrum. Meth. A 389, 81 (1997)

[3] C.R. Harris, K.J. Millman, S.J. van der Walt, R. Gommers, P. Virtanen, D. Cournapeau, E. Wieser, J. Taylor, S. Berg, N.J. Smith et al., Array programming with NumPy, Nature 585, 357 (2020)

[4] J. Pivarski et al., Awkward Array, https://doi .org/10.5281/zenodo. 4539721

[5] J. Pivarski, P. Elmer, D. Lange, Awkward Arrays in Python, C++, and Numba, in European Physical Journal Web of Conferences (2020), Vol. 245 of European Physical Journal Web of Conferences, p. 05023, 2001.06307

[6] V. Vasilev, P. Canal, A. Naumann, P. Russo, Cling - The New Interactive Interpreter for ROOT 6, Journal of Physics: Conference Series 396, 052071 (2012)

[7] G. Reid, Thinking in PostScript (Addison-Wesley, 1990), ISBN 9780201523720, https://books . google. com/books?id=ZeRXvgAACAAJ

[8] Technical Committee X3J14, ANSI Forth Standard (1994), https://www. taygeta. com/forth/dpans.html

[9] E.D. Rather, D.R. Colburn, C.H. Moore, The Evolution of Forth, in The Second ACM SIGPLAN Conference on History of Programming Languages (Association for Computing Machinery, New York, NY, USA, 1993), HOPL-II, p. 177-199, ISBN 0897915704, https://doi.org/10.1145/154766.155369

[10] Google ProtoBuf, https://developers.google.com/protocol-buffers/

[11] Apache Thrift, https://thrift . apache.org/

[12] Apache Avro, http://avro . apache.org/

[13] Google FlatBuffers, https://google.github.io/flatbuffers/

[14] JSON, https://www . json.org/

[15] J. Blomer, P. Canal, A. Naumann, D. Piparo, Evolution of the ROOT Tree I/O, in European Physical Journal Web of Conferences (2020), Vol. 245 of European Physical Journal Web of Conferences, p. 02030, 2003.07669

[16] Apache Parquet, https://parquet . apache.org/

[17] Apache Arrow, https://arrow . apache.org/

[18] D. Beazley, Understanding the Python GIL, in PyCON Python Conference. Atlanta, Georgia (2010)

[19] Performance measurement code for this study, https://github.com/scikit-hep/ awkward-1.0/tree/1.1.0/studies/awkward-forth-performance 